\title{
Research On Inspection Technology Of Tunnel Disease Based On Panoramic Vision
}

\author{
Kegang $\mathrm{Hu}^{1, \mathrm{a}}$, Qi Chen ${ }^{2, \mathrm{~b}}$ and Yiping Tang ${ }^{3, \mathrm{c}}$ \\ ${ }^{1}$ College of Information Engineering, Zhejiang University of Technology, Hangzhou, 310023, China \\ ${ }^{2}$ College of Information Engineering, Zhejiang University of Technology, Hangzhou, 310023, China \\ ${ }^{3}$ Author to whom correspondence should be addressed; College of Information Engineering, \\ Zhejiang University of Technology, Hangzhou, 310023, China \\ a email: 18767159193@139.com, ${ }^{\mathrm{b}}$ email: chenqibob@foxmail.com, ${ }^{\mathrm{c}}$ email: typ@zjut.edu.cn
}

Keywords: Panoramic vision; Tunnel; Disease inspection; Image processing

\begin{abstract}
In order to solve the general exist problems of visual based tunnel disease inspection such as low level of automation and intelligence, using a high cost performance tunnel disease inspection device to have high precision automatic analysis and evaluation on the face of tunnel across time and space, the article designed a disease inspection technology for the whole section of tunnel. First designed a Panoramic visual sensor which can fast access the panoramic image of the whole tunnel section, and then through digital image processing technology, have high-precision automatic analysis and assessment on tunnel panoramic images, identification and classification on diseases, and provide metadata support to the analysis of the dynamic change of the developing tunnel disease across time and space. The experimental results showed that the visual based tunnel disease inspection can accurately and quickly search the tunnel diseases, automatically detect and have judgments and assessment on various defects exist in tunnel.
\end{abstract}

\section{Introduction}

The cracks and water seepage of the tunnel is two main diseases of tunnel engineering. At the beginning of the tunnel construction, less money, as part of the tunnel construction cycle short, caused many tunnels in when it was completed in a timely manner to support and formed a "naked hole tunnel".

After many years the tunnels built in, because there is no lining protection, as the time goes on, in the geological terrain conditions, climate conditions, and in the process of design, construction, operation, under the influence of various factors in the process of long-term use, appear all sorts of different degrees of cracks and water seepage, the serious influence to our country's railway train operation safety [1].

\section{Tunnel disease inspection technology based on panoramic vision hardware system}

In this paper, a kind of tunnel disease inspection technology based on panoramic vision hardware system includes: tunnel inspection car, panoramic vision sensors, lighting illuminant, the remote computer. The tunnel inspection device based on panoramic vision is shown in figure 1.

The Omni-directional vision sensor (ODVS) is a visual sensor that exclusively developed by the author of this paper. It is made by a common camera and a hyperboloid catadioptric lens, keep the hyperboloid catadioptric lens optic axis and the axis of optical axis in the same line of cameras, camera resolution is $1280 \times 720$, field range is $120^{\circ} \times 360^{\circ}$, the detailed design of ODVS in [2]. Panoramic vision sensors installed in the front of the tunnel check-out car central location, in order to get a panoramic view of the tunnel lining.

Because the dark tunnel environment, and building materials on the surface of the lining, generally it is a darker color, is not conducive to the sensor experiment, so on the Omni-directional vision sensor fixed a set of LED, so as to achieve the effect of lighting for tunnel lining; Lighting light source 
design, on the other hand, the advantages and disadvantages are also affects the success or failure of the whole part of the image capture [3].

In this paper, the experiment used the tunnel check-out car body adopts the MT-R robot, production of the Automation Technology Co., Ltd., this article only use the robot motion control interface and related hardware equipment.

The configuration of the remote computer used in this article is as follows: the Pentium 4 CPU, $2 \mathrm{~g}$ of memory, independent display card, $512 \mathrm{MB}$ of RAM, the operating system is Windows 7, reading the tunnel section of panoramic image, the unwrap of tunnel section of panoramic image, and segmentation, tunnels suspected disease image processing are realized by using Java programming.

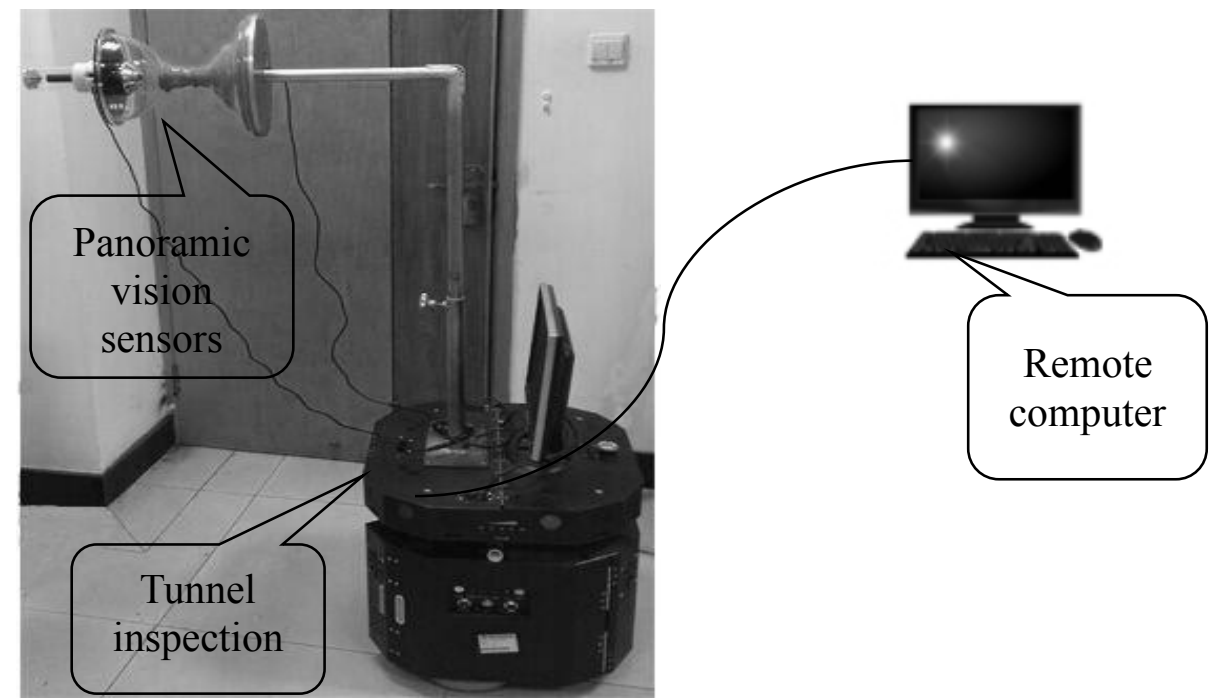

Fig.1. The tunnel inspection device based on panoramic vision

\section{Tunnel disease inspection technology based on panoramic vision software system}

System software includes: tunnel section of panoramic image data read unit, tunnel cross-section panoramic image and segmentation unit image preprocessing unit, tunnels, tunnels suspected disease suspected disease image processing unit, the tunnel diseases image features extraction unit.

Work in the field of image processing analysis system mainly includes the following steps:

\section{Tunnels suspected disease image preprocessing}

Tunnels suspected disease image preprocessing of the main work is suspected disease image enhancement of the tunnel, including gray level correction and image smoothing, the main effect of this work is to improve the image quality, for the follow-up of the tunnel suspected disease image in preparation for further processing.

This paper adopts an adaptive image histogram equalization processing method, adaptive histogram equalization to consider the location information of source image, the method based on local statistical characteristics of image pixel to pixel gray value for function transformation, transformation function has a pixel around a sub image histogram to determine the size of.

In this paper, the image smooth adopts wiener filtering method [4], the wiener filter gets minimum mean square error of the mathematical expectation value as the optimal standard.

\section{Tunnels suspected disease image processing}

Tunnels suspected disease image processing includes: image segmentation and image morphology processing image to choose the appropriate method for suspected tunnel diseases; Here, first of all, using the Otsu method [5] suspected disease image binary segmentation of the tunnel, and then to the tunnel suspected of binary image mathematical morphology processing; In mathematical morphology processing adopts opening operation to closing operation.

Opening operator is shown in formula (1):

$A^{\circ} B=(A \ominus B) \oplus B$.

Closing operator is shown in formula (2): 
Where $A$ is the original image of tunnel suspected disease, $B$ is the structural elements of the image.
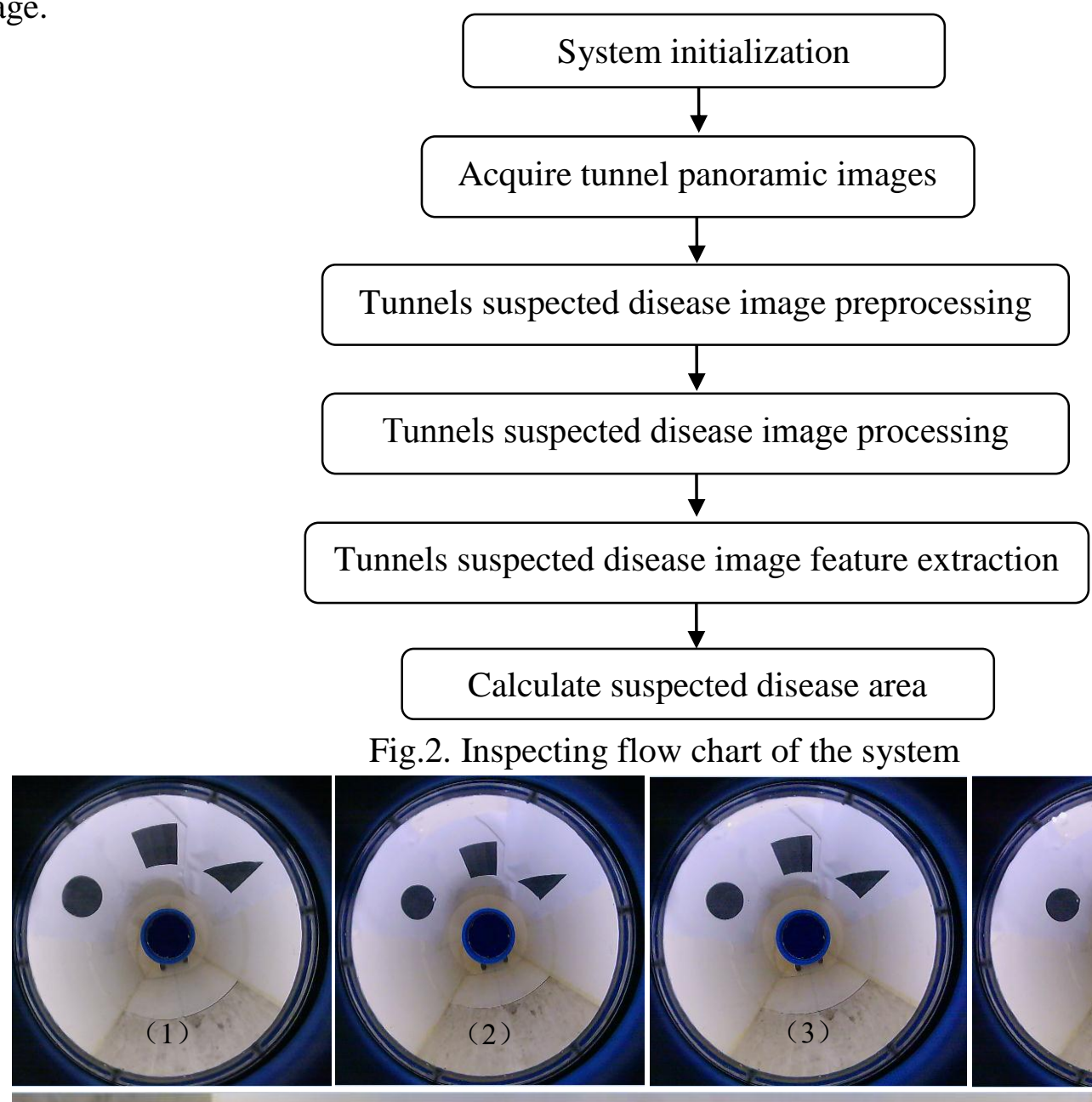

Fig.2. Inspecting flow chart of the system

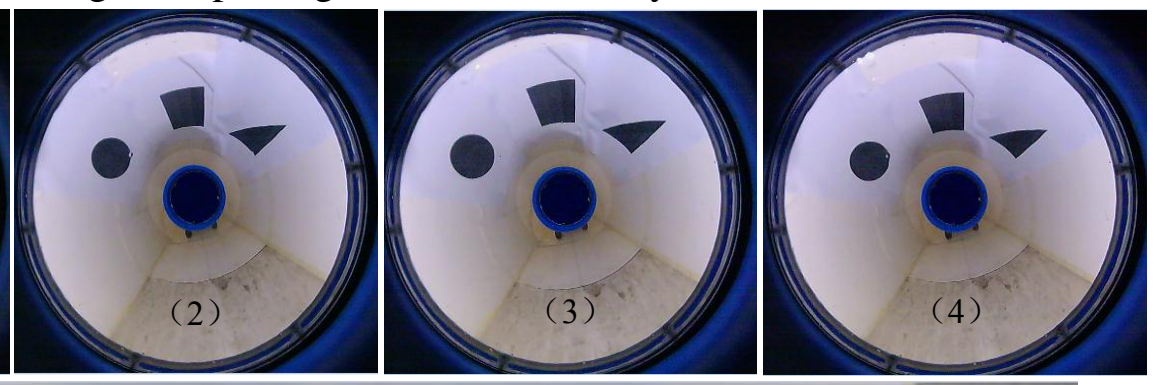

(1).a
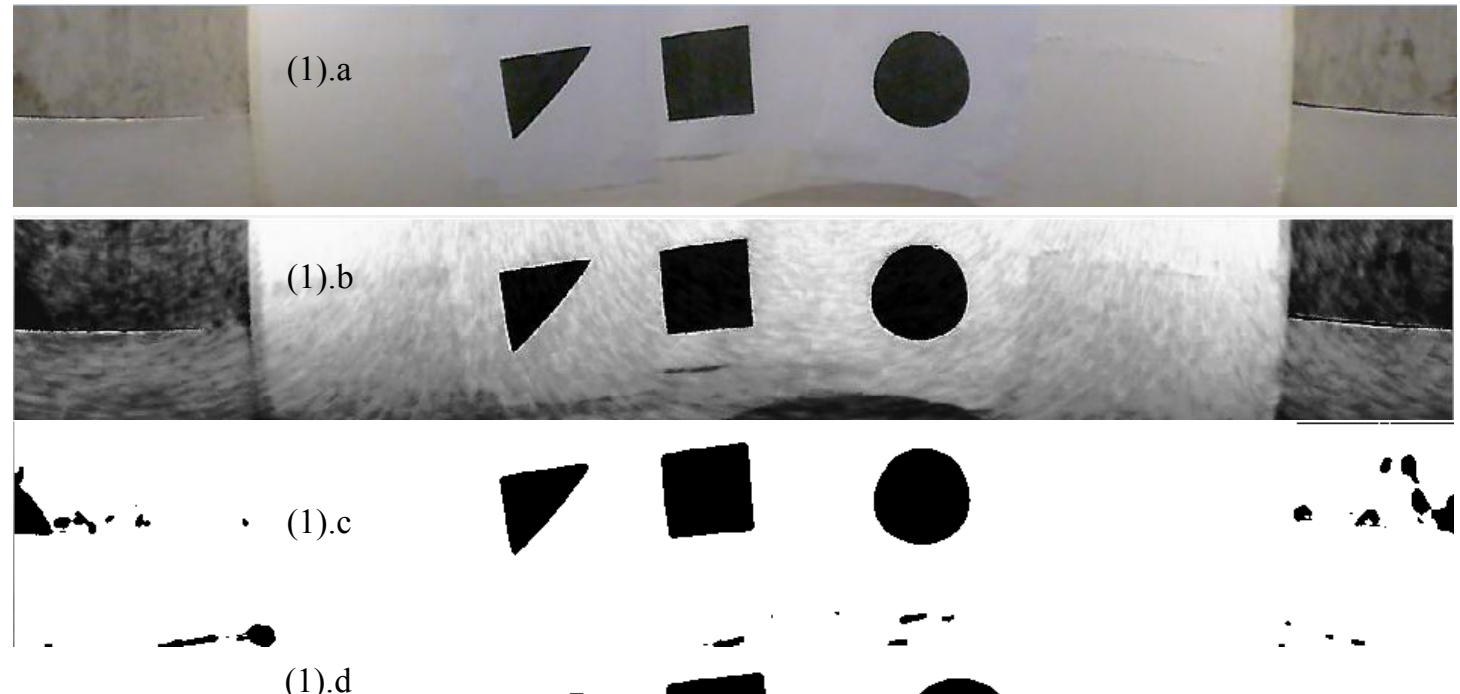

(1).d

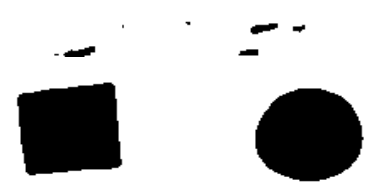

Fig.3. A set of experiments

\section{Tunnels suspected disease image feature extraction}

To recognize and distinguish the tunnel diseases from the tunnel suspected disease image, the area of the tunnel diseases can be calculated. Calculate disease area in the binary image: 


$$
A=\frac{\text { Total } D}{\text { Total Image }} \times P \text {. }
$$

Where $A$ is the tunnel disease area, Total $D$ is the pixels of tunnel disease image, Total Image is the pixels of tunnel image, $P$ is the actual size of the tunnel.

Inspecting flow chart of the system shown in figure 2 .

According to the above steps after threshold segmentation the tunnel suspected disease in binary image, there are only two kinds of pixels, disease area pixel value is 1, the background area of pixel value is 0 , which can be extracted to disease area of pixels in an image. The disease actual area can be calculated. Then we can set a certain threshold, which can determine the tunnel disease types.

\section{Experimental design and data processing}

In this paper, the design of experiments was conducted under the condition of simulated experiment environment, designs a set of experiments, and obtains a series of experiments images through the above system. There have 4 experiments images, (1).a shows the panoramic unwrap image, (1).b shows the preprocessing image, (1).c shows the thresholding image, (1).d deletes the pavement of the tunnel. Table 1 shows the data of the experiments. $\boldsymbol{A}^{*}$ is the real disease area.

Tab.1. The data of the experiments

\begin{tabular}{|l|l|l|l|l|l|l|}
\hline Number & Total Image & Total D & $\boldsymbol{P}$ & $\boldsymbol{A}\left(\mathbf{c m}^{\mathbf{2}}\right)$ & $\boldsymbol{A}^{*}\left(\mathbf{c m}^{\mathbf{2}}\right)$ & Relative error(\%) \\
\hline$(1)$ & 98272 & 2640 & 8549 & 229.7 & 228.5 & 0.53 \\
\hline$(2)$ & 98272 & 2640 & 8724 & 234.4 & 228.5 & 2.57 \\
\hline$(3)$ & 98272 & 2640 & 8653 & 232.5 & 228.5 & 1.73 \\
\hline$(4)$ & 98272 & 2640 & 8786 & 236.0 & 228.5 & 3.29 \\
\hline
\end{tabular}

\section{Summary}

This paper designs a kind of inspection technology of tunnel disease based on panoramic vision, uses the digital image processing technology, analysis and calculate the tunnel disease, develops tunnel disease inspection software. The future will be to establish tunnel physical health archives and provides effective technical support for the maintenance and completion acceptance of the tunnel.

\section{Acknowledgements}

This work was financially supported by the National Nature Science Foundation (61070134).

\section{References}

[1] Xue-zeng Liu, YunLong Sang, Yunfan Su. Tunnel seepage disease detection based on digital image processing technology $[\mathrm{J}]$. Journal of rock mechanics and engineering, 2012,S2:3779-3786.

[2] Yi-Ping Tang, Qing Wang, Jun Jiang. Design of Vertically-aligned Binocular Omnistereo Vision Sensor with Face-to-Back Configuration [J]. EURASIP Journal on Image and Video Processing, 2010: 3-12.

[3] Rui Wang. Research on Image Processing of In-vehicle Tunnel Lining Crack Detection [D]. Chengdu: Southwest Jiaotong University, 2012.

[4] Xie-hua Sun. Digital Image Processing [M]. Beijing: China Machine Press, 2010.

[5] Mark S. Nixon, Alberto S. Aguado. Feature Extraction and Image Processing, Second Edition [M]. Singapore: Elsevier Pte Ltd, 2010. 